\title{
AN APPROACH FOR OBJECT FINDING USING MOBILE ROBOTS BASED ON ACO
}

\author{
Mrs. Amita.P. Meshram ${ }^{1}$ and Mrs. Smita .R. Kapse ${ }^{2}$ \\ ${ }^{1}$ Department of Computer Technology, Y.C.C.E Hingna Road,Nagpur.16 India. \\ amitameshram@gmail.com \\ ${ }^{2}$ Department of Computer Technology, Y.C.C.E Hingna Road, Nagpur.16 India. \\ kawadesmita@gmail.com
}

\begin{abstract}
In this paper, we propose Ant Colony Optimization (ACO) for mobile robot. This paper describes the analysis and design of a new class of mobile robots. These small robots are intended to be simple and inexpensive, and will all be physically identical, thus constituting a homogeneous team of robots. They derive their usefulness from their group actions, performing physical tasks and making cooperative decisions as a Coordinated Team. This method based on heuristic concept is used to obtain global search. Since the proposed method is very efficient, thus it can perform object finding very quickly. In the process of doing so, we first use ACO to obtain the shortest obstructed distance, which is an effective method for arbitrary shape obstacles.
\end{abstract}

\section{KEYWORDS}

Mobile robots, ant colony algorithm, object finding, beacon.

\section{INTRODUCTION}

The Computer systems consist of heterogeneous software/hardware/network components with a range of capacities and availability, and with applicability in varied contexts. As computer systems become increasingly large and complex, their dependability and autonomy play a critical role at supporting next-generation science, engineering, and commercial applications [9][10]. They provide computing services to large pools of users and applications, and, thus, can be used for any optimization process.

One algorithm that has been successful in solving optimization problems is the ant colony optimization (ACO) algorithm. The ACO algorithm is a population-based metaheuristic inspired from the natural behaviour of trail laying and following by ants. While the ant colony optimization algorithm has been applied successfully to numerous optimization problems there are only limited applications in the image processing field. Only recently, researchers began to apply ACO to image processing tasks such as object identification, edge detection and image segmentation as well as texture classification. Path planning is a key step in the control of mobile robot. And the quality of path influences the efficiency of mobile robot. So designing an efficient path planning algorithm is essential.

The remaining of this paper is organized as follows. Section 2 provides a brief description of the work conducted with ant algorithms in object finding task. Section 3 gives an overview of ant colony optimization. Section 4 presents the proposed ant-based approach. In section 5, the working is described. Section 6 presents Results. Finally, conclusions are described in section 7. 
International Journal of Distributed and Parallel Systems (IJDPS) Vol.2, No.6, November 2011

\section{RELATED WORK}

Presently, there are many algorithms for path planning, such as Artificial Potential Field (APF) [11], Fuzzy Logic (FL) [12], Neural Networks (NN) [13], Genetic Algorithm (GA) Ant Colony Optimization (ACO) and so on. However, these algorithms can't reach an ideal solution separately in complex dynamic environment. For example, APF usually gets into local minimum easily. Fuzzy logic offers a possibility to mimic expert human knowledge. However, when the input increases, the reasoning rules would expand rapidly, and the computation would mount up exponentially. Neural network has the capability to learn from existing knowledge, but the knowledge representation is very difficult. GA is an evolutionary algorithm, and able to resolve composition optimization problems. But it updates the good individuals entirely and doesn't have exploited the characteristics of the path solution space. ACO is fit for the combination optimization problems, such as path planning, but it can't be applied in dynamic environment.

Considering the success ACO has in solving complex optimization problems and the few initial applications of ACO to image processing, this study was motivated to apply the ant colony methodology to the object finding problem. The goal of the study was to design an algorithm to perform optimization, as well as explore the applicability of the ant algorithm to such problem, particularly in comparison with established methods. Our ultimate goal is to achieve full automation of the following processes: (i) Simultaneous tracking of multiple mobile robots, (ii) Recognition of individual and colony behaviours, (iii) Acquisition of new single and multiagent behaviour models, (iv) application of the acquired models to multi-agent software and robotic systems.

In real system, mobile robot always knows a lot of information about static obstacles in environment, so we can exploit the information to improve the algorithm efficiency. Therefore, mobile robot can plan the global route before moving [2]. This paper combines the characteristics of $\mathrm{ACO}$ and $\mathrm{APF}$, and proposes a path planning approach in dynamic environment that integrates the global planner and local planner. The basic idea is that, ACO is used to plan the global route based on static environment information, and then APF is utilized to program the local route. In this paper, the pheromone information obtained from ACO is exploited to prevent it from getting into local minimum [4].

\section{Ant Colony Optimization}

Ant colony optimization (ACO) is a technique that optimizes problems through guided search of the solution space [3]. The ACO metaheuristic was originally designed to solve the Travelling Salesman problem, but has since been extended to a wide range of applications. The ACO algorithm is inspired from the natural trail following behaviour of ants. As an ant travels through paths, it drops pheromone, which then influences the selection of paths by the other ants. The deposits work like a positive feedback by reinforcing good choices. The first ACO algorithm was the Ant System. Since then, other implementations of the algorithm have been developed. The ACO metaheuristic involves solution construction on a graph. Many ants travel through the solution space adding solution components to partial solutions until they reach a complete solution. The selection of the components depends on the pheromone content of the paths and a heuristic evaluation [1].

\section{Proposed Methodology}

Similar to the gravitational forces in spatial self-organization, each robot's position in the behavioral space is affected by activation or inhibition forces coming from teammates (and obstacles). Figure 1 shows the behavioural modules of an Army-ant robot. As seen in the figure 
1 , robot behaviours are affected by other agent's status / behaviours as well as their own sensory data. The lines define the activation caused by a specific behaviour module or sensory data.

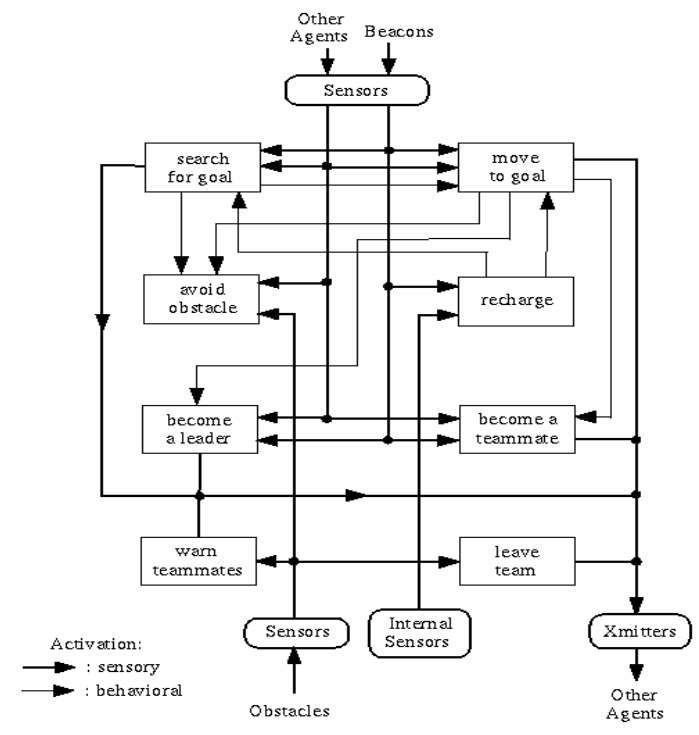

Figure1. Behaviour modules of the Mobile robots

For example, avoid-obstacle behaviour is induced by search and move-to-goal behaviours, and is also affected by sensor data signalling the presence of other agents and/or obstacles. On the other hand, the overall behaviour of the team being affected by each individual behaviour to some degree, the size of the team and the environment changes as the robots operate. Therefore, Army-ant robot behaviours have to be modelled (if possible) as a complex dynamic system with continuously changing parameters. Although there are artificial intelligence methods to define decision systems by using behavioural models, the behavioural model of an Army-ant team will be much more complicated than a single agent case. Proposed system will use different methodology for different process of execution.

\subsection{Wireless transition}

All robots are controlled through remote controller which generates different Radio frequencies to communicate with different robot. A pair of transmitter and the receiver is used for each robot. Each robot will receive and transmit the signals.

\subsection{Colour identification}

All the robots having same task that is object identification, so we defined object identification using object's colour. In proposed system robot will identify the object's colour using IR circuit which will generate different values for different colour like RGB. 


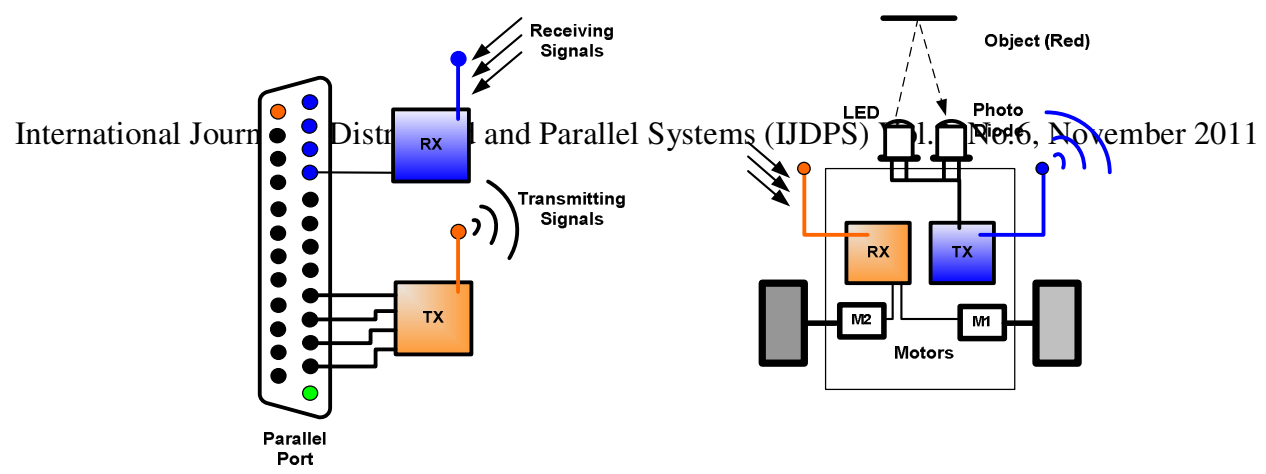

Figure 2. Single Ant structure with transmitter and receiver

In this work, a method for finding the optimal path from an initial point to a final one in a previously defined static search map is presented based on Ant Colony Optimization method. The proposed algorithm supports the avoidance of dynamic obstacles that is, once the optimal path is found and the robot starts navigating, if the robot's route is interrupted by a new obstacle that was sensed at time $t$, it will recalculate an alternative optimal path from the actual robot position in order to surround this blocking object and reach the goal as shown in figure 3 .

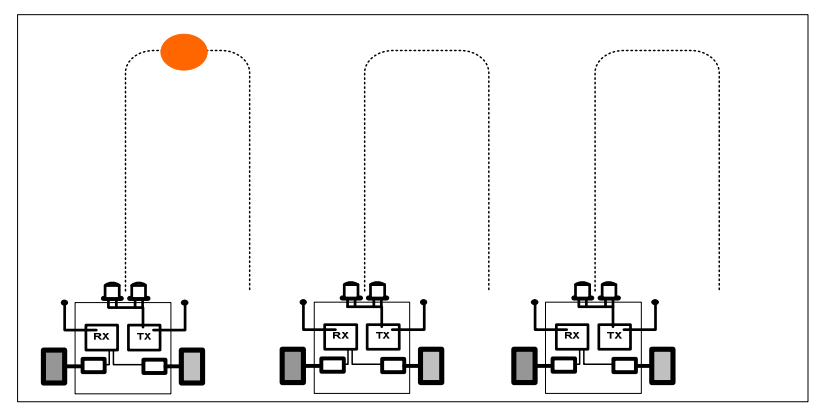

Figure 3. Maze structure with virtual path

\subsection{Algorithm based on ACO}

Mode 1: Search object (Master)

Thread (main) Random Run

Start loop till object found (sub thread)

$\mathrm{A}=\mathrm{Get}$ number Randomly $(0,2)$ : For direction range 0,2

$\mathrm{B}=$ Get number Randomly $(1000,5000)$ :

For Run Delay range $1 \mathrm{sec}$ to $5 \mathrm{sec}$

Select case A

Case 0 for Forward

On Data pin $(0 * 0378,6)$

Case 1 for Left

On Data port $(0 * 0378,10)$

Case 2 for Right

On Data port $(0 * 0378,5)$

End select delay (B)

On Data port $(0 * 0378,0)$ : For Reset

Hardware (data port)

Save/Record A\& B in text file i.e. FP :

(FP will become path for Slave Ant)

Continue loop 
International Journal of Distributed and Parallel Systems (IJDPS) Vol.2, No.6, November 2011

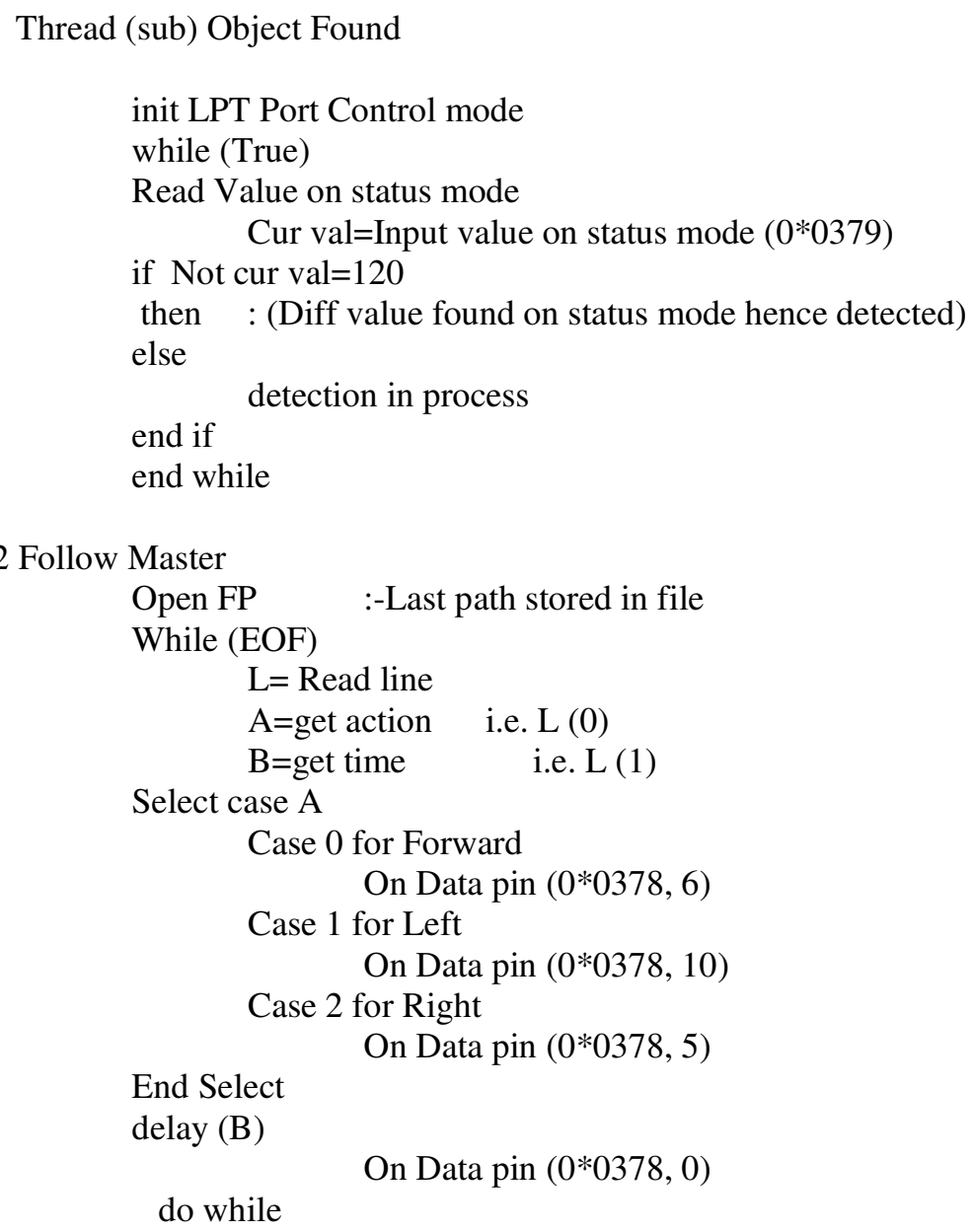

\section{BASIC WORKING}

In this work, a method for finding the optimal path from an initial point to a final one in a previously defined static search map is presented based on Ant Colony Optimization method. The proposed algorithm supports the avoidance of dynamic obstacles; that is, once the optimal path is found and the robot starts navigating, if the robot's route is interrupted by a new obstacle that was sensed at time $t$, it will recalculate an alternative optimal path from the actual robot position in order to surround this blocking object and reach the goal.

In the object finding path planning research field, heuristic methods have demonstrated to outperform classical approaches gaining popularity in the last many years. Several ideas have been proposed to overcome the complex nature of this NP-Complete problem. Ant Colony Optimization algorithms are heuristic methods that have been successfully used to deal with this kind of problems. In the decision making process which is influenced by the existing distance between the source and target nodes moreover the ants can remember the visited nodes. The selection of the optimal path relies in the criterion of a Fuzzy Inference System. The path planner application has two operating modes, one is for virtual environments, and the second one works with a real mobile robot using wireless communication. Both operating modes are global planners for plain terrain and support static and dynamic obstacle avoidance. 


\section{EXPERIMENTAL RESULTS}

After Execution the desired output screens are as follows.

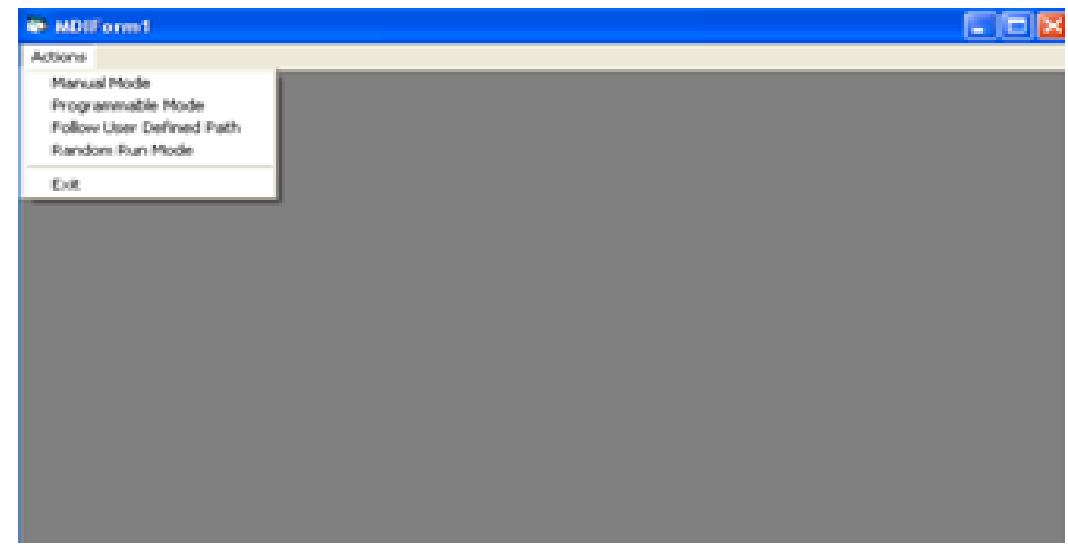

Figure 4. MDI Form with Four Modes

Manual Mode

Programmable Mode

Follow User Defined Mode

Random Run Mode

Exit

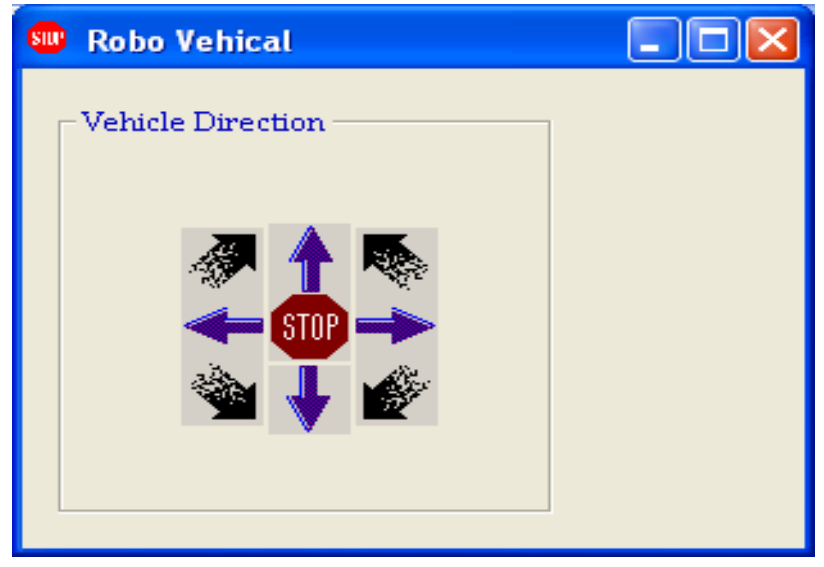

Figure 5. Robot Vehical

In this Manual Mode it gives the various directions to the Ant Robot.

Select case A

Case 0 for Forward

On Data pin $(0 * 0378,6)$

Case 1 for Left

On Data pin $(0 * 0378,10)$

Case 2 for Right

On Data pin $(0 * 0378,5)$ 
International Journal of Distributed and Parallel Systems (IJDPS) Vol.2, No.6, November 2011

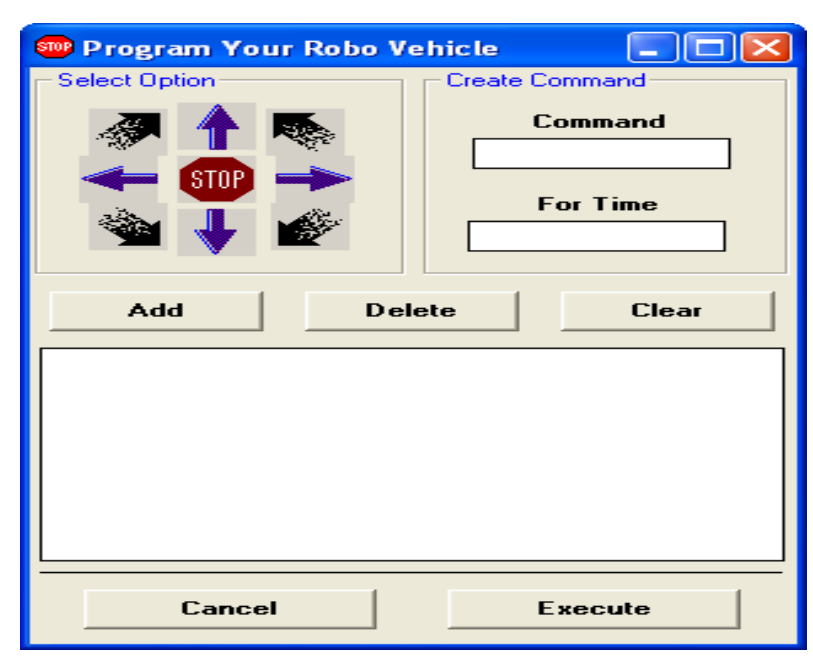

Figure 6. Programmable Mode

Processor gives the command to Ant robot for movement i.e. going in various directions. System adds the various direction and time to reach for the particular destination.

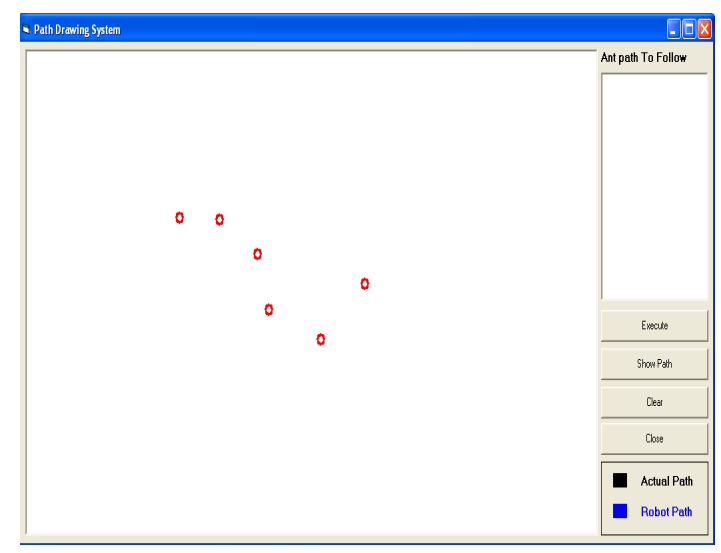

Figure 7.Follow user define mode

As per points given by user, system detects the position of those points. It means it detect the $\mathrm{x}$, y co-ordinates of that points and locate that points. With the help of given points the system draw path. And that path will be followed by Ant Robot. 
International Journal of Distributed and Parallel Systems (IJDPS) Vol.2, No.6, November 2011

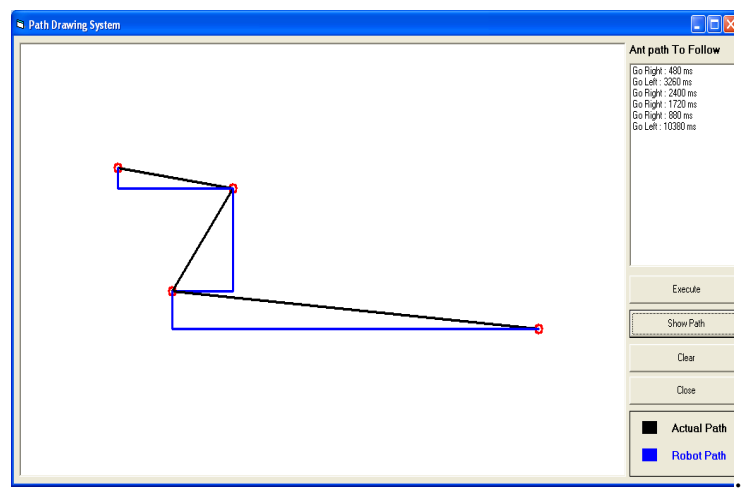

Figure 8. Path Drawing System

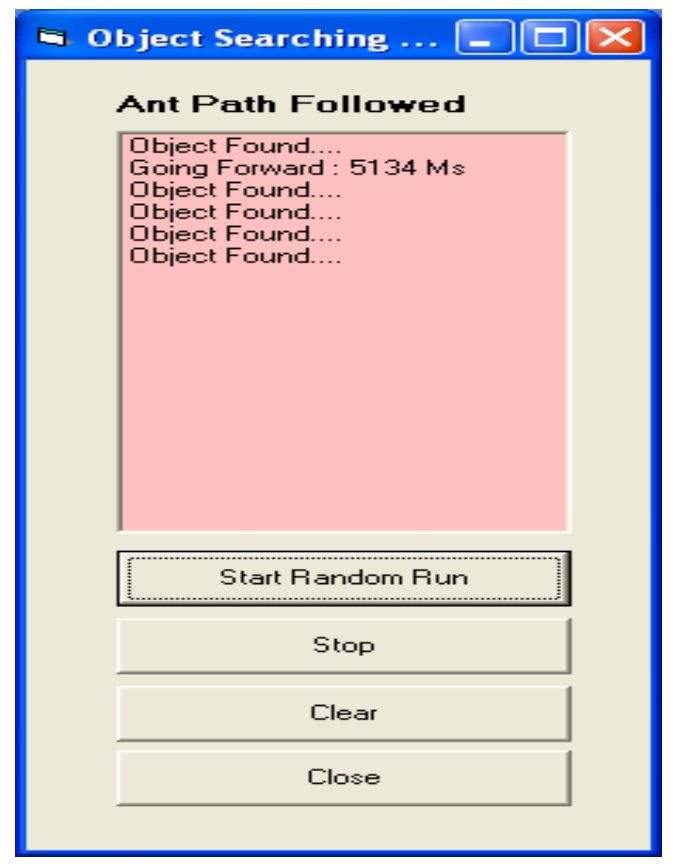

Figure 9. Random Run Mode

Ant Robot Search objects randomly. When Ant Robot (master) found that object it stops searching. Show the message "Object Found". That path will be recorded by server and server transmits that path for another ant robot (slave). While travelling, master Ant robot will send continuous signal to server. Server will record it to keep master ant robot track. This track will used to guide next slave ant robot to find the object. Software is for to auto finding that path by Ants, where the object is placed. In this Random Run Mode, let the user draw defined path and robot will follow it. 
International Journal of Distributed and Parallel Systems (IJDPS) Vol.2, No.6, November 2011

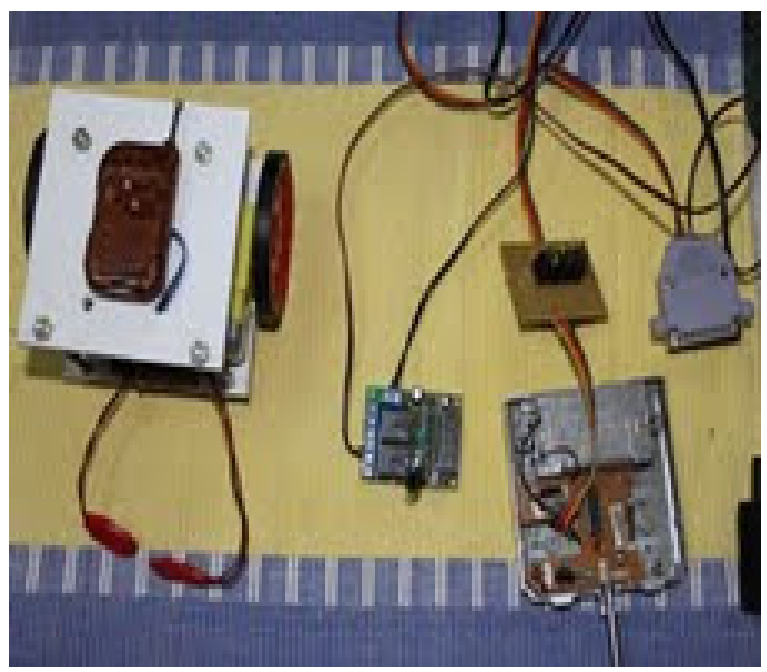

Figure 10. Single Ant Robot Hardware

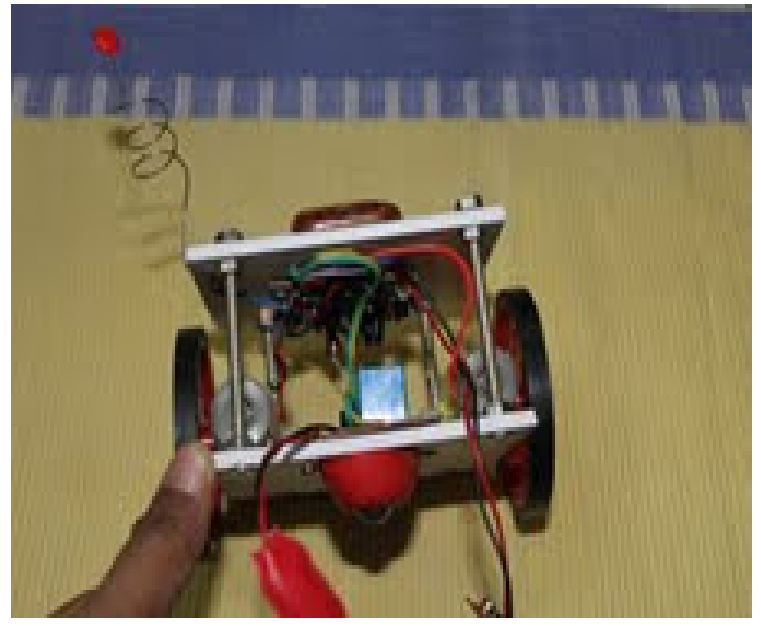

Figure 11. Autonomous ant Robot

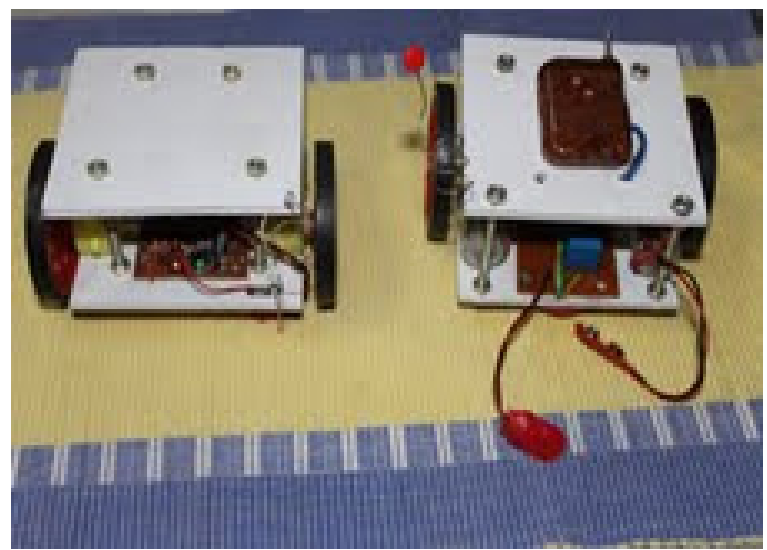

Figure 12. Autonomous ant Robot 
International Journal of Distributed and Parallel Systems (IJDPS) Vol.2, No.6, November 2011

\section{CONCLUSION}

This paper presents a framework for controlling multiple mobile robots connected by communication networks. This framework provides a technique to control coordinated systems using mobile agents. Instead of physical movement of multiple robots, mobile software agents migrate from one robot to another so that they can minimize energy consumption in aggregation. In order to avoid excessive energy consumption, we employed mobile software agents to locate robots scattered in a field, and make them autonomously determine their moving behaviours by using an algorithm based on the Ant Colony Optimization (ACO).

\section{REFERENCES}

[1] J. Pena, , M.G. Hinchey, R. Sterritt, "Towards Modeling, Specifying and Deploying Policies in Autonomous and Autonomic Systems Using an AOSE Methodology", ease,pp. 37-46, Third IEEE International Workshop on Engineering of Autonomic \& Autonomous Systems(EASE'06), 2006.

[2] First International Conference on Advances in Computer-Human Interaction obstacle. “Avoidance Path Planning for Mobile Robot Based on Multi Colony Ant Algorithm”.Nguyen Hoang Viet, Ngo Anh Vien, SeungGwan Lee, and TaeChoong Chung Artificial Intelligence Lab, Department of Computer Engineering, School of Electronics and Information, Kyunghee University 0-7695-30876-9/08 \$25.00 @ 2008 IEEE DOI 10.1109/ACHI.2008.42

[3] M. Dorigo and T. Stützle, Ant Colony Optimization. Cambridge, Massachusetts: The MIT Press, 2004.

[4] W. Truszkowski, M. Hinchey, J. Rash, C. Rouff, "NASA's swarm missions: the challenge of building autonomous software", IT Professional, vol. 6, no. 5, 2004, pp. 47-52.

[5] Xie, M., Dai, Y.S., Poh, K. L. Computing Systems Reliability: Models and Analysis, Kluwer Academic Publishers: New York, NY, U.S.A, 2004.

[6] Zou, X., Ramamurthy, B., Magliveras, S., Secure Group Communication over Data Networks, Springer, ISBN: 0-387-22970-1. 2004.

[7] W. F. Truszkowski, M. G. Hinchey, J. L. Rash, C. A. Rouff, "Autonomous and Autonomic Systems: A Paradigm for Future Space Exploration Missions", Systems, Man and Cybernetics, Part C, IEEE Transactions on, vol. 36, no. 3 2006. Proceedings of the 2nd IEEE International Symposium on Dependable, Autonomic and Secure Computing (DASC'06) 0-7695-2539-3/06 $\$ 20.00$ (C) 2006 Authorized licensed

[8] Y.S. Dai, M. Xie, K. L. Poh, "Markov renewal models for correlated software failures of multiple types" IEEE Transactions on Reliability, 2005 vol. 54, no. 1, pp. 100-106.

[9] Y.S. Dai, T. Marshall, X. H .Guan, "Autonomic and dependable computing: moving towards a model-driven approach", Journal of Computer Science, Accepted for publication 2006.

[10] Zhiye Li, Xiong Chen and Wendong Xiao. A New Motion Planning Approach Based on Artificial Potential Field in Unknown Environment. PDCAT 2004, LNCS 3320, pp. 376-382, 2004.International Journal of Information Technology, Vol.12, No.3, 2006

[11] Ni Bin, Chen Xiong, Zhang Liming, and Xiao Wendong. Recurrent Neural Network for Robot Path Planning. PDCAT 2004, LNCS 3320, pp. 188-191, 2004.

[12] Luo Xiong, Fan Xiao-ping, Yi Sheng, Zhang Heng. A Novel Genetic Algorithm for Robot Path Planning in Environment Containing Large Numbers of Irregular Obstacles. ROBOT, Vol.26, No.1, Jan.2004.

[13] Shuhua Liu, Yantao Tian, Jinfang Liu. Multi Mobile Robot Path Planning Based on Genetic Algorithm. Proceedings of the 5th World Congress on Intelligent Control and Automation, 2004, 4706-4709 


\section{Mrs. Amita.P. Meshram}

She received the $\mathrm{BE}$ degree in Computer Technology from Nagpur University. She receives her ME degree in Wireless Communication and Computing from Rastrasant Tukdoji Maharaj Nagpur University. Her research interest are in wireless network,ad-hoc network and robotics.

\section{Mrs. Smita R. Kapse}

She received the $\mathrm{BE}$ degree in Computer Technology from Nagpur University in 2002. She receives her $\mathrm{ME}$ degree in Wireless Communication and Computing from Rastrasant Tukdoji Maharaj Nagpur University in 2009. Her research interest are in wireless network, ad-hoc network and robotics. She is a member of ACM
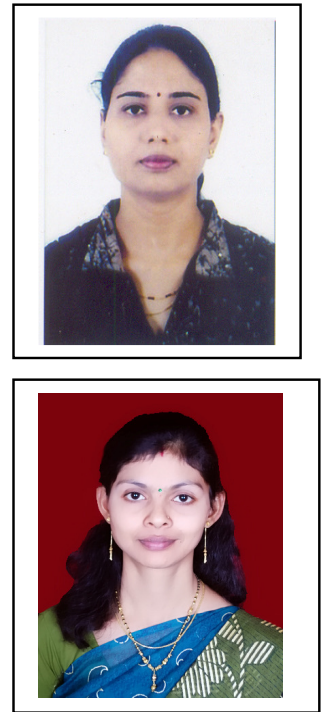